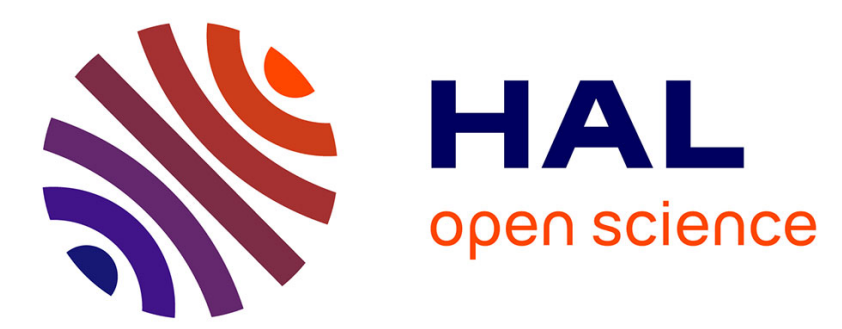

\title{
Ultimate strength properties of fiber-reinforced composites
}

\author{
François Hild, Pascal Feillard
}

\section{To cite this version:}

François Hild, Pascal Feillard. Ultimate strength properties of fiber-reinforced composites. Reliability Engineering and System Safety, 1997, 56 (3), pp.225-235. 10.1016/S0951-8320(97)00094-X . hal02342283

\section{HAL Id: hal-02342283 \\ https://hal.science/hal-02342283}

Submitted on 31 Oct 2019

HAL is a multi-disciplinary open access archive for the deposit and dissemination of scientific research documents, whether they are published or not. The documents may come from teaching and research institutions in France or abroad, or from public or private research centers.
L'archive ouverte pluridisciplinaire HAL, est destinée au dépôt et à la diffusion de documents scientifiques de niveau recherche, publiés ou non, émanant des établissements d'enseignement et de recherche français ou étrangers, des laboratoires publics ou privés. 


\title{
Ultimate strength properties of fiber- reinforced composites*
}

\author{
François Hild \& Pascal Feillard \\ Laboratoire de Mécanique et Technologie, ENS Cachan/CNRS/Université Paris VI, 61 avenue du Président Wilson, F-94235 \\ Cachan Cedex, France
}

\begin{abstract}
The volume effect and stress heterogeneity effect (i.e., the effect of loading type) on the ultimate strength are analyzed for fiber-reinforced composites. The main failure mechanisms are assumed to be fiber breakage and fiber pull-out. Depending on the load redistribution around a broken fiber, two different regimes can be obtained. The results are applied to the prediction of ultimate strengths of $\mathrm{SiC}$ fiber-reinforced composites subjected to tension, pure flexure and three-point flexure.
\end{abstract}

\section{INTRODUCTION}

In spite of the fact that Fiber-Reinforced Composites (FRCs) are made of brittle fibers, the composites can exhibit a fracture resistance behavior $[1,2]$. The origin of the fracture resistance is the consequence of the statistical failure strength of fibers (i.e., all the fibers do not break at the same load level and they can be subject to multiple breakage) and of sliding which occurs between broken fibers and the surrounding matrix. The non-linearity of a stress/strain curve close to the ultimate point is one of the indications of multiple fragmentation of fibers [3]. In this paper we study the case where the ultimate strength of FRCs is dictated by fiber breakage and fiber pull-out. The multiple fragmentation of fibers depends upon the statistical nature of fiber breakage as well as the load redistribution around a fiber break. Two extreme regimes (or modes) can be exhibited as described in an earlier analysis by Guicer and Gurland [4]. The first, referred to as a global load sharing regime assumes that the load carried by a fiber at failure is equally shared by all the unbroken fibers. This hypothesis was also used to model dry fiber bundles [5-7]. Global load sharing is an important assumption that allows us to relate the fragmentation analysis of a single fiber composite to fiber breakage and fiber pull-out in a multi-fiber composite. On the other hand, a local load sharing regime assumes that there exists a weakest zone in the material where the onset of a

* This work was partially supported by the Advanced Research Projects Agency through the University Research Initiative, ONR Contract NO0014-12-J-1808. failure mechanism leads to the failure of the composite (e.g., a fiber adjacent to a previously broken fiber breaks because of the stress concentration induced by the initially broken fiber [8] bundle pull-out [9]). Because of localized load shedding from a broken fiber on to the nearest neighboring fibers, localized breaks induce the final failure of the composite.

The assessment of ultimate strengths of FRCs is mainly based upon the assumption of a constant interfacial sliding stress in the case of ceramic matrices [10-13] or ductile matrices [14-16] in the global load sharing regime although the same assumption can be used to describe bundle pull-out [9]. A constant interfacial sliding stress is also assumed to study multiple fragmentation taking place in polymeric- as well as ceramic-matrix composites [17-20]. To carry out the analysis, an elementary cell can be considered whose size, the recovery length, is directly related to the longest fiber that can be pulled out and cause a reduction in load carrying capacity $[10,13]$. In the case of polymeric matrices other models have been used [21] based upon more refined mechanical models of the interfacial behavior [22]. The latter studies deal with the distribution of fragment length and therefore one relevant length to consider is the average fragment length. This length can be used to derive ultimate strengths of FRCs, regardless of the details of the mechanical behavior of the interface. From a design point of view, it is important not only to evaluate the ultimate tensile strength of FRCs but to model the stress/strain response of the composite so that the capacity of the components subjected to multiple loadings can be estimated [23]. We focus our attention on determining the strength of rectangular 
beams made of unidirectional composites subjected to a combination of tension and flexure so that closed-form solutions can be obtained. In the first part it is shown that the ultimate tensile strength can be derived by considering an elementary cell of size equal to the average fragment length. The second part is concerned with a constitutive law derived in tension and compression to analyze the pure flexural loadings discussed in the third part. In pure flexure, the failure mode is assumed to be identical to that in tension (i.e., no compressive mode is considered in the present analysis). The fourth part establishes the interaction between tensile and flexural loadings and the fifth is concerned with the study of the ultimate strength in three-point flexure. Experimental data are compared with predictions and the two load transfer regimes are discussed.

\section{ULTIMATE TENSILE STRENGTH}

In this section, expressions of the ultimate tensile strength of FRCs are derived. The key mechanism leading to final failure of the considered FRC is assumed to be fiber breakage. This mechanism is usually characterized by the fact that a fiber undergoes multiple fragmentation until final fracture. The matrix contribution is supposed to be negligible compared to that of the fibers. Depending on the analyzed system, different models are used to describe the behavior of the fiber/matrix interface. The simplest assumption is to consider a constant interfacial shear stress $\tau$ $[24,25]$. Other models have been proposed $[26,22]$ in which the debonding propagation is based upon a shear lag analysis limited by an interfacial shear strength and a Coulomb friction law which models the load transfer along the debond length. In both cases, expressions of the average fragment length are derived numerically $[17,27]$ as well as analytically $[19,28]$. The latter quantity will be used to assess the ultimate tensile strength of FRCs.

In the first part a global load sharing regime is supposed to occur. By assuming that the fibers do not interact, a single fiber system is then representative of the whole composite behavior $[18,11,16]$. Let us consider an elementary cell of size equal to the average fragment length $\vec{L}$. The average fragment length is defined as the ratio of the total composite length $L$ divided by the average number of fiber breaks $\bar{N}(T)$ in a single fiber

$$
\widetilde{L}(T)=\frac{L}{\bar{N}(T)}
$$

where $T$ is the reference stress equal to the stress level in an unbroken fiber for the same macroscopic strain $\bar{\epsilon}$. Furthermore, the average stress $\bar{\sigma}$ is related to the stress field along the fibers in the elementary cell $\sigma_{F}(T, z)$ by

$$
\bar{\sigma}(T)=\frac{f}{\bar{L}(T)} \int_{-\bar{L}(T) / 2}^{\bar{L}(T) / 2} \sigma_{F}(T, z) \mathrm{d} z
$$

where $z$ is the current position along the fiber direction, and $f$ the fiber volume fraction. Since the reference stress $T$ is directly proportional to the macroscopic strain $\bar{\epsilon}$, the ultimate tensile strength is reached when

$$
\frac{\partial \bar{\sigma}\left(T=T_{u}\right)}{\partial T}=0
$$

where the reference stress corresponding to the ultimate tensile strength is denoted by $T_{u}$. If we assume that the interfacial shear resistance is modeled by a constant shear stress $\tau$, the average stress is given by

$$
\bar{\sigma}(T)=f T\left[1-\frac{L_{R}(T)}{2 \bar{L}(T)}\right]
$$

where $L_{R}$ is the so-called recovery length. The latter is related to the reference stress by

$$
L_{R}(T)=\frac{R T}{\tau}
$$

where $R$ is the fiber radius. The recovery length refers to twice the longest fiber that can be pulled out and cause a reduction of the load carrying capacity (Fig. 1).

It is worth noting that eqn (4) can be used in the case of a constant interfacial shear stress when the ratio $L_{R}(T) / \bar{L}(T)$ is approximated by the cumulative failure probability $P_{F}(T)$ of a piece of composite of length $L_{R}(T)$ [13]. As long as the interaction between
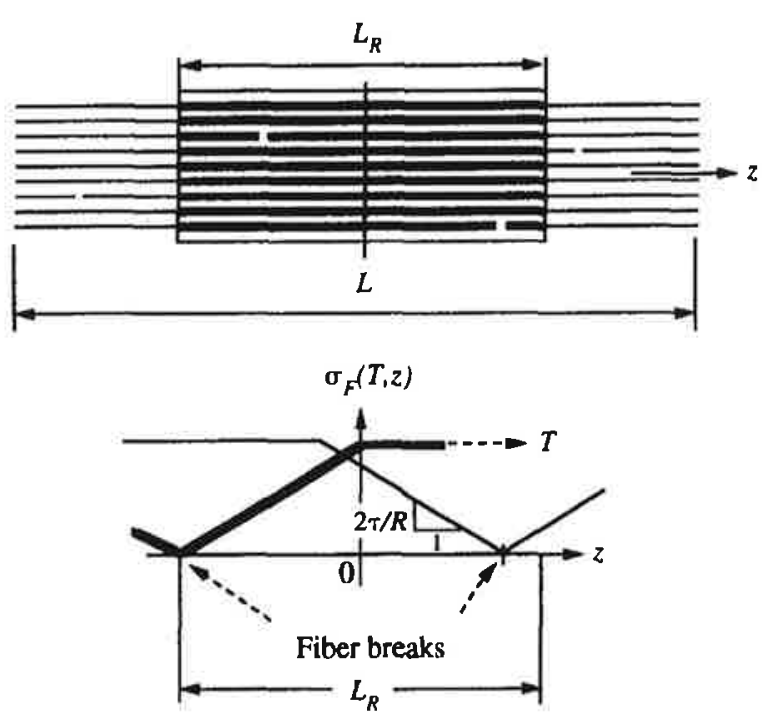

Fig. 1. Distribution of the fiber stress field $\sigma_{F}(T, z)$ for a given value of the reference stress $T$ in the case of a constant interfacial shear stress $\tau$. Two different fibers are considered. 
fiber breaks is negligible (i.e., $L_{R}(T) / \bar{L}(T) \ll 1$ ), the evolution of the average fragment length is given by $[10,13]$

$$
\frac{L_{R}(T)}{\bar{L}(T)}=1-\exp \left[-\left(\frac{T}{S_{c}}\right)^{m+1}\right]
$$

where $m$ is the Weibull modulus [29] modeling fiber failure, and $S_{c}$ is the characteristic strength [17] defined as

$$
S_{c}=\left(\frac{S_{0}^{m} L_{0} \tau}{R}\right)^{\frac{1}{m+1}}
$$

where $S_{0}$ is the scale parameter and $L_{0}$ the corresponding gauge length of the Weibull law modeling fiber failure. Equation (6) neglects the fragments of size less than or equal to $L_{R}(T)$ (i.e., it is assumed that there are no over-lapping recovery regions). This assumption is not very strong since the strain levels at the ultimate point are significantly lower than those at fiber breakage saturation [13]. A first order solution of the ratio $L_{R}(T) / \bar{L}(T)$ is found to be

$$
\frac{L_{R}(T)}{\bar{L}(T)} \cong:\left(\frac{T}{S_{c}}\right)^{m+1}
$$

and the corresponding ultimate strength $\bar{\sigma}_{U T S}$ is given by

$$
\bar{\sigma}_{U T S} \cong f S_{c}\left(\frac{2}{m+2}\right)^{\frac{1}{m+1}} \frac{m+1}{m+2} .
$$

This expression is rigorously identical to that found by Curtin [10]. Equation (9) shows that the characteristic strength is the scaling stress needed to derive the ultimate tensile strength. Furthermore, eqn (9) is valid provided the composite length $L$ is greater than the recovery length at the ultimate $L_{R}\left(T_{u}\right)$, which is proportional to the reference length $\delta_{c}$ defined by [19]

$$
\delta_{c}=\frac{R S_{c}}{\tau}
$$

The characteristic strength $S_{c}$ can be reinterpreted as the average strength of a fiber of length $\delta_{c}$. When the previous condition is not met, the ultimate tensile strength is length-dependent and the dry fiber bundle strength $[6,7]$ is a good approximation of the ultimate tensile strength $[30,13]$

$$
\frac{\overline{\boldsymbol{\sigma}}_{U T S}}{f S_{0}} \cong\left(\frac{L_{0}}{L m e}\right)^{\frac{1}{n}}
$$

Equations (9) and (11) show that the volume effect is different from that of purely brittle materials or FRCs exhibiting a local load sharing regime for which the ultimate tensile strength, defined as the average failure stress, $\bar{\sigma}_{F T}$ is proportional to $V^{-1 / m_{s}}$ for any composite volume $V$

$$
\frac{\bar{\sigma}_{s}}{S_{s}}=\left(\frac{V_{s}}{V}\right)^{\frac{1}{m_{s}}} \Gamma\left(1+\frac{1}{m_{s}}\right)
$$

where $\Gamma($.$) is the Euler function of the second kind$ (also called gamma function), $S_{s}$ is the Weibull scale parameter, $V_{s}$ is the reference volume and $m_{s}$ is the Weibull shape parameter modeling the onset of localized failure.

If debonding propagation is based upon a shear lag analysis limited by an interfacial shear strength, and a Coulomb friction law modeling the load transfer along the debond length $[26,22]$, the exclusion zones where no additional fiber breakage is possible are not as easily determined as previously (since the recovery length is of statistical nature). However, the general formalism of eqns (2) and (3) still applies. Instead of one quantity modeling the interfacial behavior, i.e., $\tau$, two quantities are used, viz. the friction coefficient $\mu$ defining the shear stress level $\tau_{f}=-\mu \sigma_{r r}$ in the debond zone, which is dependent upon the radial stresses $\sigma_{r r}$, and the maximum shear strength $\tau_{d}$. The expression of the friction stress $\tau_{f}$ shows that the Poisson effect as well as the residual stresses are accounted for in this model [31]. An expression of the average fragment length has to be derived numerically [31] for any combination of $\tau_{d}, \mu$ and initial residual stresses in the composite. The distribution of fragments can be approximated by a Weibull distribution and the average fragment length is therefore given by

$$
\beta \bar{L}(T)=\beta L_{1}(T) \Gamma\left(1+\frac{1}{m_{1}(T)}\right)
$$

where $L_{1}(T)$ and $m_{1}(T)$ are the Weibull scale and shape parameters, respectively. The parameters model the fragment length distribution, are identified from the numerical simulations and depend upon the reference stress level $T$. The parameter $\beta$ is a function of the elastic properties of the matrix and the fiber as well as the fiber volume fraction [32]

$$
\beta=\sqrt{\frac{2 G_{m}}{E_{f} R^{2} \ln \left(\frac{\bar{R}}{R}\right)}}
$$

where $E_{f}$ is the Young's modulus of the fibers, $G_{m}$ is the shear modulus of the matrix, $2 \bar{R}$ is the average distance between fibers. When fiber breakage saturates, the parameters $L_{1}(T)$ and $m_{1}(T)$ saturate as well (see Fig. 2 in the case of a unidirectional $\mathrm{SiC} / \mathrm{LAS}$ composite). The numerical computations are performed on a single filament representative of the composite. The results of Fig. 2 were obtained with an analysis of a fiber containing 500,000 elements of length $20 \mu \mathrm{m}$ whose strength is randomly distributed 


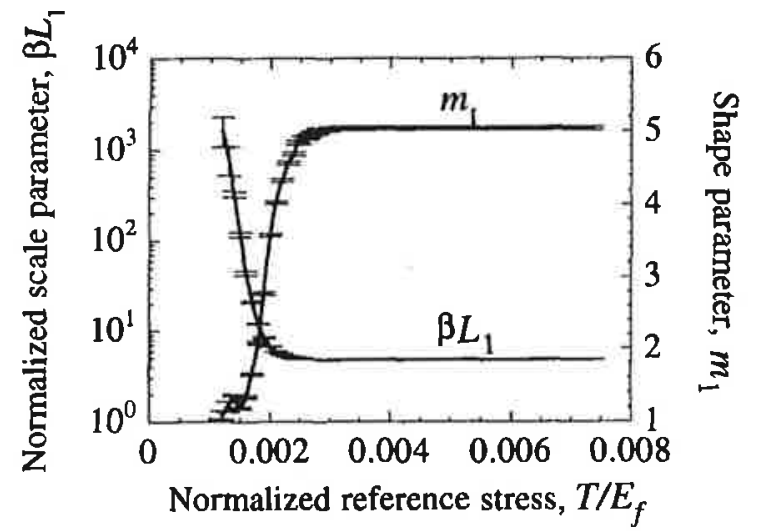

Fig. 2. Shape and scale parameters of the fiber fragment distribution as a function of normalized reference stress for a SiC/LAS composite $\left(f=0.5, \quad \beta=50 \mathrm{~mm}^{-1}, \quad S_{0}=\right.$ $\left.1500 \mathrm{MPa}, L_{0}=10 \mathrm{~mm}, m=4, \mu=0.16, \tau_{d}=50 \mathrm{MPa}\right)$.

according to a Weibull law. Because of the statistical distribution of fragment lengths, the debond lengths are also of statistical nature. A more detailed presentation of the numerical analysis used to predict the present results can be found in $[31,33]$.

The fragmentation model can be utilized to model matrix cracking as well as fiber breakage. If matrix cracking saturates, the interfacial shear stress $\tau_{f}$ does not evolve significantly when the reference stress increases. Under these circumstances, the results obtained by a model with a constant shear strength are expected to be close to those obtained by the above-discussed model. In particular the evolution of the ultimate tensile strength as a function of the composite length is identical as shown in Fig. 3 when $\tau=\tau_{f}$. Each simulation of Fig. 3 is the result of 1,000 realizations for the same fiber length.

Before saturation, however, the previous results do not apply and a constant shear strength hypothesis can be a crude approximation of the actual interfacial behavior. In the following, the ultimate tensile

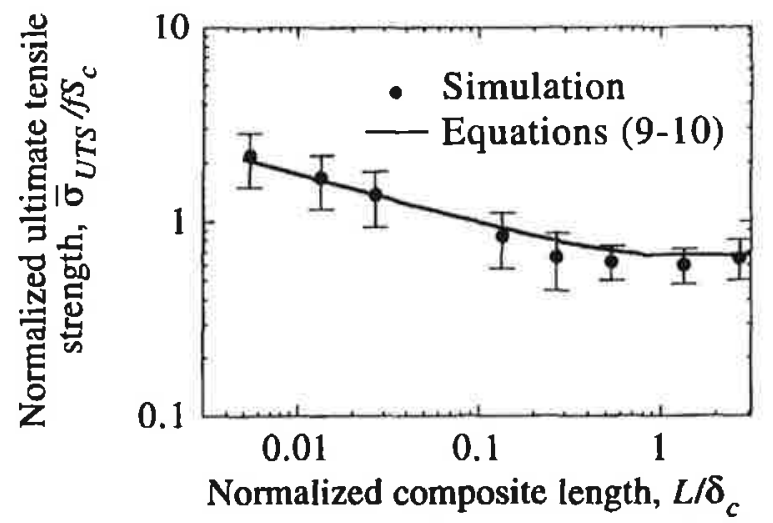

Fig. 3. Normalized ultimate tensile strength of a $\mathrm{SiC} / \mathrm{LAS}$ composite as a function of normalized composite length $\left(f=0.5, S_{\mathrm{c}}=2500 \mathrm{MPa}, m=4\right)$.

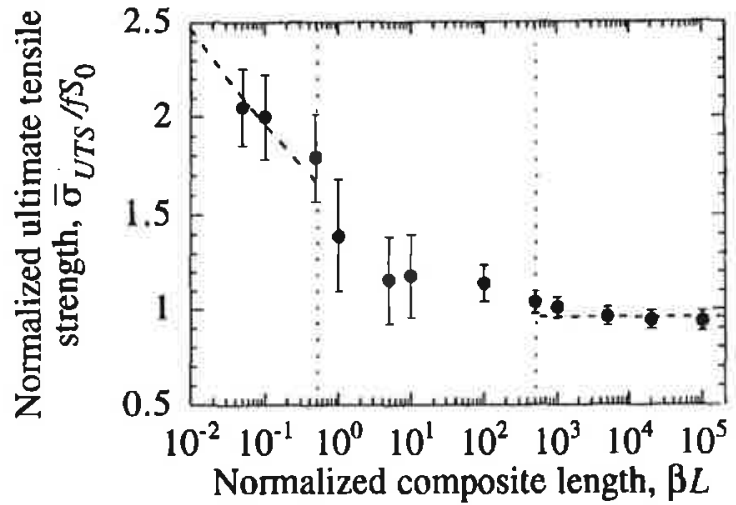

Fig. 4. Normalized ultimate tensile strength of an $E$ Glass/Epoxy composite as a function of normalized composite length $\left(\beta=10 \mathrm{~mm}^{-1}, f S_{0}=900 \mathrm{MPa}, L_{n}=10 \mathrm{~mm}\right.$, $\left.m=10, \mu=0.9, \tau_{d}=80 \mathrm{MPa}, R=5.5 \mu \mathrm{m}\right)$.

strength of an Epoxy matrix reinforced by E Glass fibers is analyzed. Figure 4 shows the evolution of the ultimate tensile strength as a function of the composite length. Each simulation of Fig. 4 is again the result of 1,000 realizations for the same fiber length. Three different regimes can be exhibited.

First, for very small composite lengths (i.e., $\beta L<0.5)$ the first break is fatal to the composite and the whole composite length containing a fiber break is debonded (see Fig. 5). In that case a constant interfacial shear stress hypothesis is admissible. Since the composite length is less than the characteristic length $\left(\delta_{c} \beta \cong 1\right)$ a length-dependent regime is expected and actually observed. The length dependence is shown to be proportional to $L^{-0.1}$ in accordance with $L^{-1 / m}$ when $m=10$ (value of the Weibull shape parameter of $E$ Glass fibers).

Second, for large composite lengths (i.e., $\beta L>500$ ) the onset of debonding signals final failure of the composite (see Fig. 5). The number of breaks is not on the order of unity as in the case of small composite

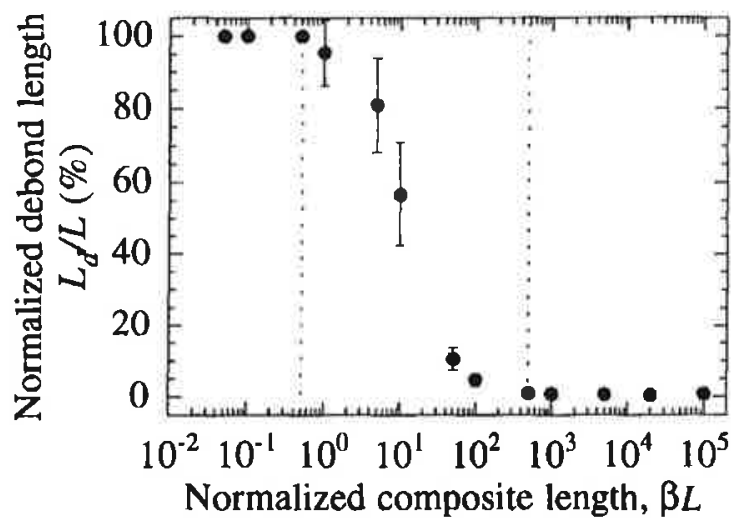

Fig. 5. Normalized debond length of an E Glass/Epoxy composite as a function of normalized composite length $\left(\beta=10 \mathrm{~mm}^{-1}, f S_{0}=900 \mathrm{MPa}, L_{0}=10 \mathrm{~mm}, m=10, \mu=0.9\right.$, $\left.\tau_{t l}=80 \mathrm{MPa}, R=5.5 \mu \mathrm{m}\right)$. 
lengths. According to the Cox model [32], the onset of debonding is given by the condition [31]

$$
2 \tau_{d}=T_{u} \beta R \tanh \left(\frac{\beta \bar{L}\left(T_{u}\right)}{2}\right) .
$$

When the average fiber fragment length $\bar{L}\left(T_{u}\right)$ is greater than $3 / \beta$, the previous condition can simplified to become $2 \tau_{d} \cong T_{u} \beta R$. This criterion is deterministic so that the ultimate tensile strength can be expressed as

$$
\bar{\sigma}_{U T S}=f T_{u}\left[1-\frac{\tanh \left(\frac{\beta \bar{L}\left(T_{u}\right)}{2}\right)}{\frac{\beta \bar{L}\left(T_{u}\right)}{2}}\right] .
$$

It is worth noting that the format of eqn (16) is identical to that of eqn (4). Furthermore, when $\beta \bar{L}\left(T_{u}\right)>100$ the previous expression can be simplified so that the ultimate tensile strength can be approached by

$$
\bar{\sigma}_{U T S} \cong f T_{u}
$$

In the present case, the ultimate tensile strength is equal to $0.96 f S_{0}$. This value is in good agreement with the numerical simulations shown in Fig. 4. Third, for composites of intermediate lengths (i.e., $0.5<\beta L<$ 500 ) the onset of debonding is not fatal to the composite (see Fig. 5). On the other hand, there is no complete debonding so none of the two previous regimes is relevant. Moreover, the number of fiber breaks increases as the composite length increases. In the case of the present simulations, the number of breaks for intermediate lengths was less than 10. Lastly, the ultimate tensile strength decreases with the total length to approach the length-independent regime when the length of the composite increases (see Fig. 4).

These simulations show that the features exhibited by a model assuming a constant interfacial shear stress can be obtained by different models even when matrix cracking is not involved. However there is (are) some additional regime(s) in-between. Moreover the lengthindependent regime can be explained by different reasons as shown by the two ultimate strength studies within the global load sharing framework.

\section{CONSTITUTIVE EQUATION}

In this section the behavior of FRCs is studied from a macroscopic point of view. Therefore we will only consider the average stress, or macroscopic stress $\bar{\sigma}$ and the corresponding macroscopic strain $\bar{\epsilon}$, or, equivalently, the reference stress $T / E_{f}$. The constitutive equations will be derived using the microscopic approach of Section 2. It is worth remembering that a constitutive equation modeling gradual fiber breakage can be written only in the global load sharing regime for which the composite behavior is lengthindependent.

In the following, it is assumed that the stress/strain behavior can be characterized by a series expansion of the macroscopic stress $\bar{\sigma}$ as a function of the reference stress $T$ modeling the gradual degradation of the material (i.e., fiber breakage, debonding and pull-out)

$$
\frac{\bar{\sigma}(T)}{f S_{c}}=\frac{T}{S_{c}}+\sum_{n \geq 1} \psi_{n}\left(\frac{T}{S_{c}}\right)^{\beta(n)}
$$

where $\psi_{n}$ are the coefficients of the series expansion, and $\beta(n)$ is a linear combination of the power $n$. Equation (18) will be used later to predict the ultimate flexural strength.

If the ultimate point is reached when $T=T_{u}=A S_{c}$, where $A$ is a constant, the ultimate tensile strength can be rewritten as

$$
\frac{\bar{\sigma}_{U T S}}{f S_{c} A}=1+\sum_{n \geq 1} \psi_{n} A^{\beta(n)-1}
$$

For example, in the case of a constant interfacial shear stress, the parameters in eqn (18) are such that $\psi_{n}=-\delta_{m n} / 2$, where $\delta_{m n}$ denotes the Kronecker symbol, $\beta(n)=\delta_{m n}(m+2), S_{c}$ is given by eqn (7) and $A=(2 /(m+2))^{m+1}$. Another application can be found in [13].

The constitutive eqn (18) is only valid in the tensile part, and does not take into account matrix cracking since the initial Young's modulus is assumed to be the Young's modulus of the unbroken fibers $f E_{f}$. In the compressive part we assume that the behavior is unaltered upon loading and is given by the behavior of the virgin material. Therefore the compressive behavior is defined by the Young's modulus of the composite $\bar{E}$. No compressive failure mechanism is considered herein. A damage variable, $D_{1}$, is introduced to measure the difference in Young's modulus in tension $\left(T / S_{\mathrm{c}} \ll 1\right)$ and compression

$$
D_{1}=1-\frac{f E_{f}}{\bar{E}} .
$$

In the next sections, this constitutive law will be used to determine ultimate strengths in pure and three-point flexure.

\section{ULTIMATE FLEXURAL STRENGTH}

This section is devoted to the determination of the ultimate strength of rectangular beams under pure flexure by making use of the model derived in tension and compression. The beam is made of one layer whose fibers are aligned along the $z$-direction. It is 


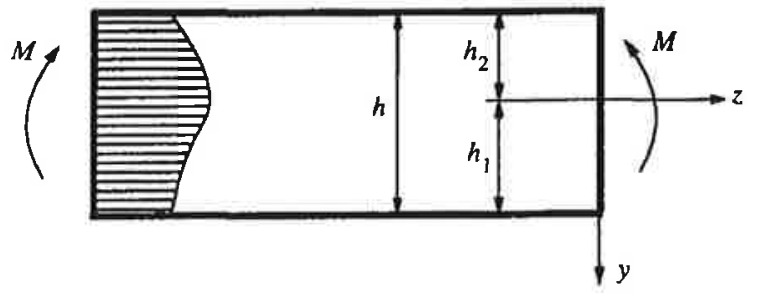

Fig. 6. Definition of the beam geometry in the case of pure flexure.

assumed that there is a sufficient number of fibers in the width of the beam so that a global load sharing hypothesis is made for each height $y$. The Bernoulli kinematic condition [34] leads to

$$
\bar{\epsilon}=\mathscr{R} y
$$

where $\mathscr{R}$ is the curvature, and $y$ the height ordinate measured from the neutral axis (see Fig. 6).

Using eqn (18), we obtain the variation of the normalized tensile stress

$$
\bar{\Sigma}=Y+\sum_{n \geq 1} \psi_{n} Y^{\beta(n)}
$$

with

$$
\bar{\Sigma}=\frac{\bar{\sigma}}{f S_{c}} \text { and } Y=\frac{E_{f}}{S_{c}} \mathscr{R} y \text {. }
$$

The position of the neutral axis $h_{1}$ (see Fig. 6) is determined from the force balance equation. The ultimate flexural strength is reached when $\bar{\sigma}\left(h_{1}\right)=$ $\bar{\sigma}_{U T S}$ and the position of the neutral axis is given by

$$
\frac{h}{h_{1}}=1+\sqrt{\left(1-D_{1}\right)\left\{1+2 \sum_{n \geq 1} \frac{\psi_{n} A^{\beta(n)-1}}{\beta(n)+1}\right\}} .
$$

Using the moment equation, the ultimate flexural strength $\bar{\sigma}_{U F S}$ is given by

$$
\begin{aligned}
\frac{\bar{\sigma}_{U F S}}{f S_{c} A}= & 2\left(\frac{h_{1}}{h}\right)^{2}\left\{\frac{1}{1-D_{1}}\left(\frac{h}{h_{1}}-1\right)^{3}\right. \\
& \left.+1+3 \sum_{n \geq 1} \frac{\psi_{n} A^{\beta(n)-1}}{\beta(n)+2}\right\} .
\end{aligned}
$$

The ratio of the ultimate flexural strength to the ultimate tensile strength is then given by

$$
\frac{\bar{\sigma}_{U F S}}{\bar{\sigma}_{U T S}}=\frac{2\left(\frac{h_{1}}{h}\right)^{2}\left\{\frac{1}{1-D_{1}}\left(\frac{h}{h_{1}}-1\right)^{3}+1+3 \sum_{n \geq 1} \frac{\psi_{n} A^{\beta(n)-1}}{\beta(n)+2}\right\}}{1+\sum_{n \geq 1} \psi_{n} A^{\beta(n)-1}} .
$$

Equation (25) shows that the ultimate strength ratio $\bar{\sigma}_{U F S} / \bar{\sigma}_{U T S}$ is independent of the composite length, but depends upon the damage parameter $D_{1}$ in addition to the parameters $\psi_{n}, \beta(n)$ and $A$. This result constitutes a second difference with a weakest link hypothesis for which the average strength ratio
$\bar{\sigma}_{F F} / \bar{\sigma}_{F T}$ for the same volume loaded in pure flexure and in tension is given by

$$
\frac{\overline{\boldsymbol{\sigma}}_{F F}}{\overline{\boldsymbol{\sigma}}_{F T}}=\left[2\left(m_{s}+1\right)\right]^{\frac{1}{m_{s}}}
$$

where $\bar{\sigma}_{F F}$ is the average failure stress in pure flexure. To illustrate the previous results, a constant interfacial shear strength is assumed in the framework of global load sharing. By using the approximations to derive eqn (8), the position of the neutral axis is given by

$$
\frac{h}{h_{1}}=1+\sqrt{\left(1-D_{1}\right) \frac{(m+1)(m+4)}{(m+2)(m+3)}}
$$

and the ultimate flexural strength $\bar{\sigma}_{U F S}$ becomes

$$
\begin{aligned}
\frac{\bar{\sigma}_{U F S}}{f S_{c}}= & 2\left(\frac{h_{1}}{h}\right)^{2}\left(\frac{2}{m+2}\right)^{\frac{1}{m+1}}\left[\frac{1}{1-D_{1}}\left(\frac{h}{h_{1}}-1\right)^{3}\right. \\
& \left.+\frac{(m+1)(m+5)}{(m+2)(m+4)}\right] .
\end{aligned}
$$

In Fig. 7 the evolution of the normalized flexural strength is plotted as a function of the Weibull parameter $m$ and the damage parameter $D_{1}$. The lower the value of the Weibull parameter, the higher the normalized flexural strength. On the other hand, the higher the value of the damage parameter, the higher the normalized flexural strength. These results have been obtained differently in [13] but are very close to those presented herein.

Lastly, when the only difference in the tension/compression behavior is given by the Young's modulus difference $(m \rightarrow+\infty)$ modeled by the damage variable $D_{1}$, eqns (27) and (28) show that ratio of the ultimate flexural strength to the ultimate tensile strength approaches $2 /\left(1+\sqrt{1-D_{1}}\right)$.

\section{INTERACTION BETWEEN FLEXURE AND TENSION}

In this section we will study stress states where we combine tensile and flexural loads. The beam characteristics are the same as those used in the previous section. We still assume a Bernoulli hypothesis [34]. The kinematic condition leads to a linear strain field in the beam of height $h$ such that (see Fig. 8)

$$
\overline{\boldsymbol{\epsilon}}(y)=\frac{T_{c}}{E_{f}} Y(y)
$$

with

$$
Y(y)=\frac{1+\alpha}{2}+\frac{1-\alpha}{h} y-\frac{h}{2} \leq y \leq \frac{h}{2} .
$$

The variable $y$ now denotes the height ordinate measured from the mid-plane of the beam, and the 


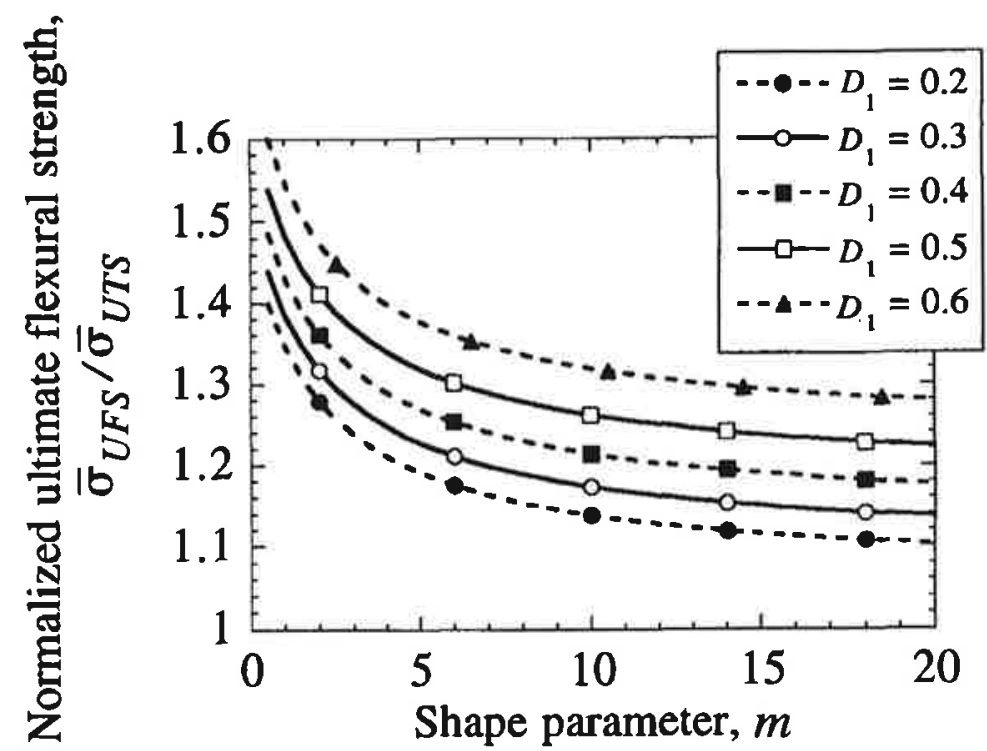

Fig. 7. Evolution of the normalized flexural strength as a function of the Weibull parameter $m$ and the damage parameter $D_{1}$.

constant $\alpha$, which is less than or equal to 1 , measures the ratio of $\bar{\epsilon}(-h / 2)$ to $\overline{\boldsymbol{\epsilon}}(h / 2)$

$$
\alpha=\frac{\overline{\boldsymbol{\epsilon}}(-h / 2)}{\overline{\boldsymbol{\epsilon}}(h / 2)} \text {. }
$$

When $\alpha$ lies between 0 and 1 then the whole beam undergoes tensile stresses, whereas negative values of $\alpha$ lead to a mixed tensile/compressive mode. The tensile strength is obtained from a force balance equation and is defined as

$$
\overline{\boldsymbol{\sigma}}_{T}=\frac{N\left[\bar{\sigma}(h / 2)=\bar{\sigma}_{U T S}\right]}{b h}
$$

where $N$ denotes the resultant force, and $b$ the width of the beam. The flexural strength is obtained from a moment balance and is defined as

$$
\bar{\sigma}_{F}=\frac{6 M\left[\bar{\sigma}(h / 2)=\bar{\sigma}_{U T S}\right]}{b h^{2}} .
$$

where $M$ denotes the resultant moment. When $\alpha$ is

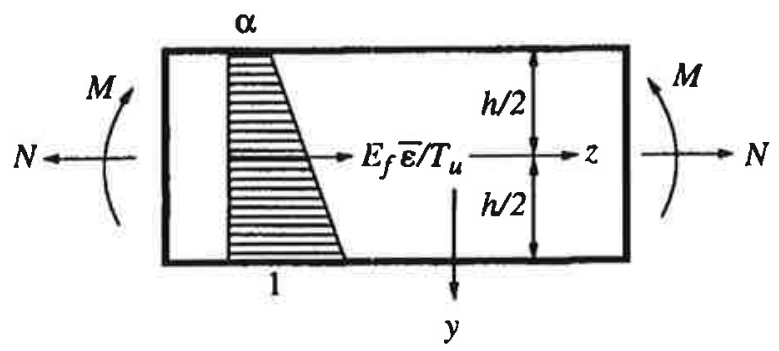

Fig. 8. Definition of the beam geometry in the case of flexure combined with tension. positive, the beam is in a pure tensile mode. Therefore the results are independent of the variable $D_{1}$. The resultant force equation yields

$\bar{\sigma}_{T}=\frac{f S_{c} A}{1-\alpha}\left\{\frac{1-\alpha^{2}}{2}+\sum_{n \geq 1} \frac{\psi_{n} A^{\beta(n)-1}}{\beta(n)+1}\left(1-\alpha^{\beta(n)+1}\right)\right\}$.

The moment equation enables us to derive the flexural strength to be

$$
\begin{aligned}
\bar{\sigma}_{F}= & -\frac{3(1+\alpha)}{1-\alpha} \bar{\sigma}_{T}+\frac{2 f S_{c} A}{(1-\alpha)^{2}} \\
& \times\left\{1-\alpha^{3}+3 \sum_{n \geq 1} \frac{\psi_{n} A^{\beta(n)-1}\left(1-\alpha^{\beta(n)+2}\right)}{\beta(n)+2}\right\} .
\end{aligned}
$$

When $\alpha$ is negative, a part of the beam is in compression. In that case the results depend on the damage variable $D_{1}$. The resultant force equation allows us to get the tensile strength

$$
\bar{\sigma}_{T}=\frac{f S_{c} A}{1-\alpha}\left\{\frac{1}{2}-\frac{\alpha^{2}}{2\left(1-D_{1}\right)}+\sum_{n \geq 1} \frac{\psi_{n} A^{\beta(n)-1}}{\beta(n)+1}\right\}
$$

and the moment equation enables us to derive the flexural strength

$$
\begin{aligned}
\bar{\sigma}_{F}= & -\frac{3(1-\alpha)}{1-\alpha} \bar{\sigma}_{T}+\frac{2 f S_{c} A}{(1-\alpha)^{2}} \\
& \times\left[1-\frac{\alpha^{3}}{1-D_{1}}+3 \sum_{n \geq 1} \frac{\psi_{n} A^{\beta(n)-1}}{\beta(n)+2}\right] .
\end{aligned}
$$

To illustrate the previous results, a constant interfacial shear strength is assumed. By using the approximations discussed to derive eqn (8), the 
following results are obtained. When $\alpha$ is positive, the resultant force equation leads to

$\overline{\boldsymbol{\sigma}}_{T}=\frac{f S_{c}}{1-\alpha}\left(\frac{2}{m+2}\right)^{\frac{1}{m+1}}\left[\frac{1-\alpha^{2}}{2}-\frac{1-\alpha^{m+3}}{(m+2)(m+3)}\right]$

and the moment equation

$$
\begin{aligned}
\bar{\sigma}_{F}= & -\frac{3(1+\alpha)}{1-\alpha} \bar{\sigma}_{T}+\frac{2 f S_{c}}{(1-\alpha)^{2}}\left(\frac{2}{m+2}\right)^{\frac{m}{m+1}} \\
& \times\left[1-\alpha^{3}-\frac{3\left(1-\alpha^{m+4}\right)}{(m+2)(m+4)}\right] .
\end{aligned}
$$

When $\alpha$ is negative, the resultant force equation allows us to get the tensile strength

$$
\begin{aligned}
\sigma_{T}=\frac{f S_{c}}{(1-\alpha)} & \left(\frac{2}{m+2}\right)^{\frac{1}{m+1}} \\
& \times\left[\frac{(m+1)(m+4)}{2(m+2)(m+3)}-\frac{\alpha^{2}}{2\left(1-D_{1}\right)}\right]
\end{aligned}
$$

and the moment equation enables us to derive the flexural strength

$$
\begin{aligned}
\bar{\sigma}_{F}=-\frac{3(1+\alpha)}{1-\alpha} & \bar{\sigma}_{T}+\frac{2 f S_{c}}{(1-\alpha)^{2}}\left(\frac{2}{m+2}\right)^{\frac{1+1}{m+1}} \\
\times & {\left[\frac{(m+1)(m+5)}{(m+2)(m+4)}-\frac{\alpha^{3}}{1-D_{1}}\right] . }
\end{aligned}
$$

In Fig. 9 an interaction diagram is plotted when $m=4$, and $D_{1}=2 / 7$ for a constant interfacial shear strength. The interaction diagram is also compared to a linear prediction: in that particular case a straight line describes very well the interaction line (see dotted line in Fig. 9). This is one difference when we compare to the interaction diagram of an elastic-perfectly

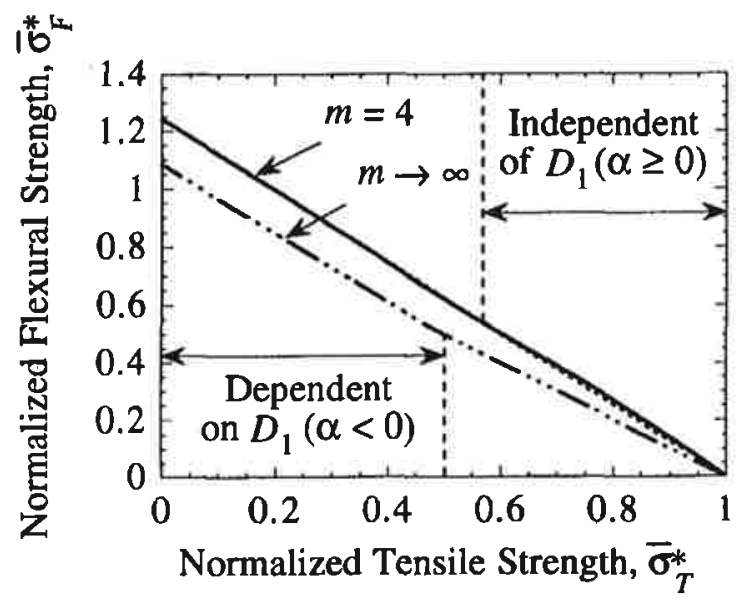

Fig. 9. Interaction diagram (solid line) between tension and flexure when $m=4$, and $D_{1}=2 / 7$ giving the normalized flexural strength $\bar{\sigma}_{F}=\bar{\sigma}_{F} / \bar{\sigma}_{U T S}$ vs the normalized tensile strength $\bar{\sigma}_{T}^{*}=\bar{\sigma}_{T} / \bar{\sigma}_{U T S}$. The dotted line gives a linear interaction. The dashed line shows the interaction diagram when $m \rightarrow+\infty$. plastic material. In that case the interaction is given by a quarter of an ellipse.

Lastly, when the only difference in the tension/compression behavior is given by the Young's modulus difference $(m \rightarrow+\infty)$ modeled by the damage variable $D_{1}$, the interaction diagram is only a function of the parameter $D_{1}$ (see Fig. 9). This last result constitutes a lower bound in the interaction diagram.

In a local load sharing regime, an interaction diagram has no meaning. However, one can study the combined effect of tension and pure flexure. The strain field defined in eqn (29) is used. The average failure stress ratio for the same volume is given by

$$
\frac{\bar{\sigma}_{F}}{\bar{\sigma}_{F T}}=\left(H_{m_{1}}\right)^{-\frac{1}{m_{w_{F}}}}
$$

where $\bar{\sigma}_{F}$ is the average failure stress under combined loading conditions, and $H_{m_{s}}$ is the Weibull stress heterogeneity factor [35] defined as

$$
\begin{gathered}
H_{m_{\mathrm{s}}}=\frac{1-\alpha^{m},}{(1-\alpha)\left(m_{s}+1\right)} \text { when } \alpha \geq 0 \\
H_{m_{s}}=\frac{1}{(1-\alpha)\left(m_{s}+1\right)} \text { when } \alpha<0 .
\end{gathered}
$$

\section{ULTIMATE THREE-POINT FLEXURAL STRENGTH}

The aim of this section is to derive the ultimate strength of rectangular beams loaded in three-point flexure. The tensile and compressive behavior follows the same hypotheses as those made in the previous sections. However, in three-point flexure the stress field profile along the fiber direction is no longer uniform. Therefore, eqn (4) cannot be used. Throughout this section, the longitudinal stress field, $\sigma(T, z)$, is assumed to be symmetric about $z=0$ and maximum at $z=0$. Depending on the position of the fiber along the $y$-axis, the stress field will vary. In the first part of the section, only a set of fibers is analyzed for which the position along the $y$-axis is the same. A global load sharing regime is assumed in a plane normal to the $y$-axis, for each value of $y$. Furthermore, the beam length is assumed to be sufficiently large with respect to the beam height and width so that the shear stresses can be neglected. For the sake of simplicity, the interfacial sliding resistance is assumed constant. The contribution of the broken fibers within the recovery length or pull-out stress, $\vec{\sigma}_{p o}$, at $z=0$ is written as

$$
\frac{\bar{\sigma}_{p o}(T)}{f}=\int_{0}^{T} \bar{\sigma}_{p}(t) \frac{\mathrm{d} P_{F}\left(t, L_{R}(t)\right)}{\mathrm{d} t} \mathrm{~d} t
$$


where $\bar{\sigma}_{p}(t)$ denotes the average stress at $z=0$ when a fiber breaks at a location $z$, and at a reference stress level $t$, and $P_{F}\left(t, L_{R}(t)\right)$ is the failure probability that a fiber break within the recovery length $L_{R}(t)$ at or below a reference stress $t$. In tension, the probability density of fracture locations is uniform, therefore $\bar{\sigma}_{p}(t)=t / 2$. On the other hand, when the stress field along the fiber direction is not homogeneous, the previous result does not apply, and one needs to determine the average fracture location to estimate the pull-out stress. The average stress $\bar{\sigma}$ applied to the composite at the plane $z=0$, is given by [10]

$\frac{\bar{\sigma}}{f}=T\left\{1-P_{F}\left(T, L_{R}(T)\right)\right\}+\frac{2 \tau}{R}(h(T)\rangle P_{F}\left(T, L_{R}(T)\right)$.

The first term of the right hand side of eqn (45) corresponds to the contribution of the unbroken fibers, and the second term is the pull-out contribution (stress $\bar{\sigma}_{p o}(T)$ ). The quantity $\langle h(T)\rangle$ represents the average pull-out length, or average fracture location in $L_{R}(T) / 2$ when the reference stress is less than or equal to $T$. The aim of the present calculation is to evaluate the average fracture location $\langle h(T)\rangle$ when the stress filed along the fiber direction is not homogeneous.

Oh and Finnie [36] derived the average fracture location of a brittle material. In that case the considered volume is constant. In the present case, the length to consider (i.e., the recovery length) is varying with the stress level measured by $T$. Therefore the results need to be generalized. By recasting the cumulative failure probability $P_{F}\left(T, L_{R}(T)\right)$ of a fiber part of length $L_{R}(T)$, useful functions are exhibited to determine the average fracture location. Under the weakest link assumption and the independent events hypothesis, the cumulative failure probability $P_{F}\left(T, L_{R}(T)\right)$ of a piece of fiber of length $L_{R}(T)$ is expressed as

$$
P_{F}\left(T, L_{R}(T)\right)=1-\exp \left[\frac{2}{L_{0}} \int_{0}^{L_{R}(T) / 2} \ln \left\{1-P_{F 0}(T, z)\right\} \mathrm{d} z\right]
$$

where $P_{F 0}(T, z)$ denotes the cumulative failure probability of a single link of length $L_{0}$ whose mid-section is located at $z$. The failure probability density is obtained by differentiation of eqn (46) with respect to $T$

$$
\frac{\mathrm{d} P_{F}\left(T, L_{R}(T)\right)}{\mathrm{d} T}=\int_{0}^{\left.L_{R} \mid T\right) / 2} 2 \Phi(T, z) \mathrm{d} z+2 \Psi(T)
$$

so that eqn (46) can be rewritten as

$$
P_{F}\left(T, L_{R}(T)\right)=\int_{0}^{T} 2\left\{\int_{0}^{L_{n}(t) / 2} \Phi(t, z) \mathrm{d} z+\Psi(t)\right\} \mathrm{d} t
$$

where $\Phi(t, z)$ corresponds to the failure probability density for a reference stress varying between $T$ and $T+d T$, of an element of length $\mathrm{d} z$ centered at $z$, and
$\Psi(T)$ is the failure probability density associated with the length increase from $L_{R}(T)$ to $L_{R}(T)+L_{R}^{\prime}(T) \mathrm{d} T$. The first term is identical to that given in $[36,37,18]$ when using a Weibull law, the second term is due to the load dependence of the recovery length $L_{R}(T)$. By using eqns (46) and (47), the expressions of the functions $\Phi$ and $\Psi$ are, respectively, given by

$$
\begin{aligned}
\Phi(T, z)= & -\left\{1-P_{F}\left(T, L_{R}(T)\right)\right\} \frac{1}{L_{0}} \\
& \times \frac{\partial}{\partial T}\left[\ln \left\{1-P_{F 0}(T, z)\right\}\right] \\
\Psi(T)=- & \left\{1-P_{F}\left(T, L_{R}(T)\right)\right\} \frac{L_{R}^{\prime}(T)}{2 L_{0}} \\
\times & {\left[\ln \left\{1-P_{F 0}\left(T, \frac{L_{R}(T)}{2}\right)\right\}\right] . }
\end{aligned}
$$

When $L_{R}(T)$ is independent of $T$, or $P_{F 0}\left(T, L_{R}(T) / 2\right)$ vanishes, $\Psi(T)$ vanishes as well, and the results given in [37] still apply in the case of a Weibull law. By dimensional analysis and by inspection of eqn (47), the average $\langle h(T)\rangle$ of the fracture location $h(T)$ for a piece of composite of length $L_{R}(T)$ at a reference stress $T$ is [38]

$$
\langle h(T)\rangle=\frac{\int_{0}^{T} 2\left\{\int_{0}^{L_{R}(t) / 2} z \Phi(t, z) \mathrm{d} z+\frac{1}{4} L_{R}(t) \Psi(t)\right\} \mathrm{d} t}{\int_{0}^{T} 2\left\{\int_{0}^{L_{R}(t) / 2} \Phi(t, z) \mathrm{d} z+\Psi(t)\right\} \mathrm{d} t} .
$$

This expression of the average fracture location does not consider the fibers that originally broke outside the recovery length but were brought into it as the load level increased. The approximation made here is worst when the stress field is constant over the whole length (i.e., pure tension). In that case, however, it can be shown that this hypothesis is not very strong [13]. Equation (45) is used for each height $y$ to determine the global stress state of the composite in three-point flexure. By studying the most loaded fibers in the tensile part, it can be shown that when the specimen length is greater than 5 times the characteristic length $\delta_{c}$, the gradient effect in three-point flexure along the fiber direction is negligible when compared to the one due to load recovery in the vicinity of a fiber break [39]. Under these circumstances the ultimate strength in threepoint flexure $\bar{\sigma}_{U 3 F}$ is on the same order of magnitude as the ultimate flexural strength $\bar{\sigma}_{U F S}$

$$
\bar{\sigma}_{U 3 F} \cong \bar{\sigma}_{U F S} \text { when } L>5 \delta_{c} \text {. }
$$

This result constitutes a major difference with materials for which a weakest link concept can be used. In that case the ratio of the ultimate strength in three-point flexure is greater than that in pure flexure. 
Table 1. Comparison between experimental (Exp.) and predicted (Pred.) ultimate strengths expressed in MPa for three composites reinforced by SiC fibers

\begin{tabular}{|c|c|c|c|c|c|c|}
\hline \multirow{2}{*}{$\begin{array}{l}\text { Loading } \\
\text { Material }\end{array}$} & \multicolumn{2}{|c|}{ Tension } & \multicolumn{2}{|c|}{ Pure Flexure } & \multicolumn{2}{|c|}{ Three-point Flexure } \\
\hline & Exp. & Pred. & Exp. & Pred. & Exp. & Pred. \\
\hline $\mathrm{SiC} / \mathrm{LAS}^{4(1)}$ & 790 & 850 & 1050 & 1080 & 1180 & 1090 \\
\hline $\mathrm{SiC} / \mathrm{C}^{41}$ & 345 & 340 & 455 & 430 & 455 & 435 \\
\hline $\mathrm{SiC} / \mathrm{Al}^{42}$ & 700 & 690 & 815 & 820 & 930 & 930 \\
\hline
\end{tabular}

When the two volumes are identical, the average failure stress ratio is given by

$$
\frac{\bar{\sigma}_{R 3}}{\bar{\sigma}_{F F}}=\left(m_{s}+1\right)^{\frac{1}{m_{s}}}
$$

where $\bar{\sigma}_{F 3}$ is the average failure stress in three-point flexure.

The previous results are applied to three composites reinforced by $\mathrm{SiC}$ fibers (Table 1). Two of the three composites have ultimate strengths following a global load sharing hypothesis (LAS and C matrices). This result is related to the fact that matrix cracking has saturated and that the interfacial shear strength is small (on the order of a few MPa). On the other hand, the aluminum matrix leads to ultimate strengths described by a local load sharing hypothesis $\left(m_{s}=7\right)$. This matrix is more sensitive to localized fiber failures and to higher values of the interfacial shear strength.

\section{CONCLUSIONS}

A unified approach to the prediction of the ultimate tensile strength of FRCs has been proposed. The key quantity to consider is the average fragment length. Expressions of the ultimate tensile strength are derived within this general framework and compared with other existing theories. In particular, in the global load sharing regime it is shown that the ultimate tensile strength is mostly length-independent. Conversely, in the local load sharing regime, the ultimate tensile strength is always length-dependent. A description of the behavior of these FRCs up to the ultimate tensile point has been derived in the framework of Continuum Mechanics.

A generalization to pure flexural modes is proposed to derive the ultimate flexural strength. In the global load sharing regime, the increase in terms of ultimate flexural strength is due to the conjunction of two phenomena: difference in elastic moduli in tension and in compression, and 'ductility' due to fiber breakage and fiber pull-out. It has been shown that the 'ductility' contributes substantially to this increase.

An interaction between tension and flexure is studied. Although some differences are noticed when compared to an elastic perfectly plastic behavior, some similar features can be shown, in particular the increase in terms of normalized ultimate strength. This case corresponds to a situation where the 'ductility' is distributed within a large region of the structure. On the other hand, when the 'ductility' is confined within a very small area of the structure, the increase in terms of the ultimate strength is expected to be less important, though the gradients along the fiber direction could play a role and increase it again.

Lastly, in the case of three-point flexure, the ultimate strength has the same order of magnitude as that in pure flexure within the framework of global load sharing. On the other hand, in the local load sharing regime, the ultimate strength in three-point flexure increases as compared with pure flexure when the two volumes are identical. The three-point flexure experiments therefore allow to discriminate the two load transfer regimes when compared with the pure flexure case. This result can be observed when ultimate strengths of LAS and C matrix composites (exhibiting a global load sharing regime) are compared with Al matrix composites (exhibiting a local load sharing regime) subjected to pure tension, pure flexure and three-point flexure.

\section{REFERENCES}

1. Netravali, A.N., Henstenburg, R.B., Phoenix, S.L. and Schwartz, P., Interfacial shear strength studies using the single-filament-composite test. Part I: Experiments on graphite fibers in epoxy. Polymer Composites, 1989, 10, $226-241$

2. Thouless, M.D., Sbaizero, O., Sigl, L.S. and Evans, A.G., Effect of interface mechanical properties on pullout in a SiC-fiber-reinforced lithium aluminium silicate glass-ceramic. Journal of the American Ceramic Society, 1989, 72, 525-532.

3. Heredia, F.E., Spearing, S.M., Evans, A.G., Mosher, P. and Curtin, W.A., Mechanical properties of carbon matrix composites reinforced with nicalon fibers. Journal of the American Ceramic Society, 1992, 75, 3017-3025.

4. Guicer, D.E. and Gurland, J., Comparison of the statistics of two fracture models. Journal of the Mechanics and Physics of Solids, 1962, 10, 365-373.

5. Pierce, F.T., Tensile tests for cotton yarns V: The 'weakest link' theorems on the strength of long and of composite specimens. Journal of the Textile Institute, 1926, 17, T355-T368.

6. Daniels, H.E., The statistical theory of the strength of 
bundles of threads. Proceedings of the Royal Society of London, 1944, 183, 405-429.

7. Coleman, B.D., On the strength of classical fibers and fiber bundles. Journal of the Mechanics and Physics of Solids, 1958, 7, 60-70.

8. Zweben, C. and Rosen, B.W., A statistical theory of material strength with application to composite materials. Journal of the Mechanics and Physics of Solids, 1970, 18, 189-206.

9. Neumeister, J.M., Bundle pullout-a failure mechanism limiting the tensile strength of continuous fiber reinforced brittle matrix composites and its implications for strength dependence on volume and type of loading. Journal of the Mechanics and Physics of Solids, 1993, 41, 1405-1424.

10. Curtin, W.A., Theory of mechanical properties of ceramic matrix composites. Journal of the American Ceramic Society, 1991, 74, 2837-2845.

11. Phoenix, S.L. and Raj, R., Scalings in fracture probabilities for a brittle matrix fiber composite. Acta Metallica Materiala, 1992, 40, 2812-2828.

12. Neumeister, J.M., A constitutive law for continuous fiber reinforced brittle matrix composites with fiber fragmentation and stress recovery. Journal of the Mechanics and Physics of Solids, 1993, 41, 1383-1404.

13. Hild, F., Domergue, J.-M., Evans, A.G. and Leckie, F.A., Tensile and flexural ultimate strength of fiberreinforced ceramic-matrix composites. International Journal of Solids and Structures, 1994, 31, 1035-1045.

14. Kelly, A. and Davies, G.J., The principles of the fiber reinforcement of metals. Metallurgy Review, 1965, 10, $1-11$.

15. Jansson, S., Mechanical characterization and modeling of non-linear deformation and fracture of a fiber reinforced metal matrix composite. Mechanics of Materials, 1991, 12, 47-62.

16. Curtin, W.A., Ultimate strength of fiber-reinforced ceramics and metals. Composites, 1993, 24, 98-102.

17. Henstenburg, R.B. and Phoenix, S.L., Interfacial shear strength studies using the single-filament-composite test, Part II: A probability model and Monte Carlo simulations. Polymer Composites, 1989, 10, 389-406.

18. Sutcu, M., Weibull statistics applied to fiber failure in ceramic composites and work of fracture. Acta Metallica, 1989, 37, 651--661.

19. Curtin, W.A., Exact theory of fiber fragmentation in single-filament composite. Journal of Materials Science, 1991, 26, 5239-5253.

20. Hui, C.Y., Phoenix, S.L., Ibnabdeljalil, M. and Smith, R.L., An exact closed form solution for fragmentation of Weibull fibers in a single filament composite with applications to fiber-reinforced ceramics. Journal of the Mechanics and Physics of Solids, 1995, 43, 1551-1585.

21. Feillard, P., Rouby, D., Désarmot, G. and Favre, J.-P., Limits of conventional micromechanical analysis of interface properties in glass/epoxy model composites. Materials Science Engineering, 1994, A188, 159-166.

22. Nairn, J.A., A variational mechanics analysis of the stresses around breaks in embedded fibers. Mechanics of Materials, 1992, 13, 131-157.

23. Hild, F., Larsson, P.-L. and Leckie, F.A., Uncoupled and fully coupled approaches to predict macro-initiation in fiber-reinforced ceramic-matrix composites. Composite Structures, 1994, 29, 365-377.
24. Kelly, A. and Tyson, W.R., Tensile properties of fibre-reinforced metals: copper/tungsten and copper/ molybdenum. Journal of the Mechanics and Physics of Solids, 1965, 13, 329-350.

25. Aveston, J., Cooper, G. A. and Kelly, A., Single and multiple fracture. In Proceedings National Physical Laboratory: Properties of Fiber Composites, IPC Science and Technology Press, Surrey, UK, 1971, pp.15-26.

26. Hutchinson, J.W. and Jensen, H.M., Models for fiber debonding and fiber pull-out in brittle composites with friction. Mechanics of Materials, 1990, 9, 139-163.

27. Feillard, P., Désarmot, G. and Favre, J.-P., A critical assessment of fragmentation test with glass/epoxy systems. Composite Science Technology, 1993, 49, 109-119.

28. Gulino, R. and Phoenix, S.L., Weibull strength statistics for graphite fibres measured from the break progression in a model graphite/glass/epoxy microcomposite. Journal of Materials Science, 1991, 26, 3107-3118.

29. Weibull, W., A statistical theory of the strength of materials. vol. 151, Roy. Swed. Inst. Engng Res., Stockholm, 1939.

30. Curtin, W.A., Strength versus gauge length in ceramic-matrix composites. Journal of the American Ceramic Society, 1994, 77, 1072-1074.

31. Feillard, P., Désarmot, G. and Favre, J.-P., Theoretical aspects of the fragmentation test. Composite Science Technology, 1994, 50, 265-279.

32. Cox, H.L., The elasticity and the strength of paper and other fibrous materials. British Journal of Applied Physics, 1952, 3, 72-79.

33. Favre, J.-P., Sigety, P. and Jacques, D., Stress transfer by shear in carbon fibre model composites, Part 2 : Computer simulation of the fragmentation test. Journal of Materials Science, 1991, 26, 189-195.

34. Timoshenko, S. P. and Goodier, J. N., Theory of Elasticity, 3rd edition, McGraw-Hill, NY, 1970.

35. Hild, F., Billardon, R. and Marquis, D., Hétérogénéité des contraintes et rupture des matériaux fragiles. $C . R$. Acad. Sci. Paris, 1992, t315, 1293-1298.

36. Oh, H.L. and Finnie, I., On the location of fracture in brittle solids-I due to static loading. International Journal of Fracture Mechanics, 1970, 6, 287-300.

37. Thouless, M.D. and Evans, A.G., Effects of pull-out on the mechanical properties of ceramic-matrix composites. Acta Metallica, 1988, 36, 517-522.

38. Hild, F., On the average pull-out length of fiberreinforced composites. C. R. Acad. Sci. Paris, 1994, t319, 1123-1128.

39. Hild, F., Ultimate strength of structures made of fiber-reinforced ceramic-matrix composites. PhD dissertation, University of California, Santa Barbara, USA, 1995.

40. Jansson, S. and Leckie, F.A., The mechanics of failure of silicon carbide fiber-reinforced glass-matrix composites. Acta Metallica Materiala, 1993, 40, 2967-2978.

41. Schwartz, S., Lee, S. and Mosher, P., Properties of silicon carbide fiber reinforced composites. In Proc. 15th Ann. Conf. Composite Materials and Structures, ACerS, Cocoa Beach, FL, 14-18 January 1994.

42. Leckie, F. A., Ultimate strength properties of SiC/Al composites. University of California, Santa Barbara, Report, 1995. 